\title{
Biportal Endoscopic Spine Surgery for Various Foraminal Lesions at the Lumbosacral Lesion
}

\author{
Dae-Jung Choi ${ }^{1}$, Ju-Eun Kim ${ }^{2}$, Je-Tea Jung ${ }^{1}$, Yong-Sang Kim ${ }^{1}$, Han-Jin Jang ${ }^{1}$, Bang Yoo $^{1}$, Il-Ho Kang ${ }^{1}$ \\ ${ }^{1}$ Spine Center, Barun Hospital, Jinju, Korea \\ ${ }^{2}$ Department of Orthopedic Surgery, Andong Hospital, Andong, Korea
}

\begin{abstract}
The stenosing foramen of L5-S1 by several degenerative diseases is one of the challenging areas on surgical approaching because of the deeper depth and steep slope in the lumbosacral junction. The floating view using unilateral biportal endoscopic spine surgery rather than docking into the Kambin's zone can make the foraminal structures seen panoramically and permit dynamic handling of various instruments without destroying the facet joint and causing iatrogenic instability. Fine discrimination of structural margins in helps of the higher magnification and gentle manipulation of neural structures just as in open spine surgery could be guaranteed using floating technique from the target structures. Selective decompression with preserving innocent structures including facet joints could relieve foraminal lesions at the L5-S1 and decrease the necessity of fusion surgery caused by wider decompression and iatrogenic instability.
\end{abstract}

Keywords: Spinal stenosis; Lumbosacral region; Minimally invasive surgery; Surgical endoscopy

\section{Introduction}

The foramen of L5-S1 developed several degenerative diseases, such as extraforaminal disc herniation, foraminal stenosis (FS), degenerative or spondylolytic spondylolisthesis, including far-out syndrome [1]. Most of them needed wider bony decompression to approach the lesions hidden under the lamina and consequently fusion surgery. Conventional endoscopic spine surgery docked into the Kambin's zone could not easily eliminate bony lesions in stenotic condition with a risk of neural irritation. Microscopic decompression using a tubular retractor surely showed a good aspect in preserving back muscle. Handling of various instruments through a long and narrow retractor under the deep and steep view, however, would be restricted and a hard working. Biportal endo- scopic spine surgery (BESS) with addressing on $2-3 \mathrm{~cm}$ apart from the pedicle could approach the L5-S1 without disturbing by the alar wing and freely handling of a scope with various angles in floating technique [2]. Safe manipulation of neural structures could be guaranteed in helps of the higher magnification and very closed proximity to the lesions. Friendly handling of various instruments used in open spine surgery could make the performances comfort and learning curve shorter.

\section{Technical Notes}

\section{Indications}

Forty-nine cases of 44 patients (five cases of both sides) of BESS for FS at the L5-S1 performed decompression using

Received Aug 30, 2017; Revised Sep 28, 2017; Accepted Oct 17, 2017

Corresponding author: Ju-Eun Kim

Department of Orthopedic Surgery, Andong Hospital, 11 Angsil-ro, Andong 36743, Korea

Tel: +82-53-420-5638, Fax: +82-53-422-6605, E-mail: dspfuture@hanmail.net 
BESS from August 2015 to December 2016 by two spine surgeons. Those included 26 cases of FS with the collapsed disc height, six cases of FS with degenerative spondylolisthesis, six cases of FS followed as adjacent segment disease after fusion surgery, five cases of FS with spondylolytic spondylolisthesis, four cases of extraforaminal lumbar disc herniation, and two cases of far-out syndrome with spur from the L5 transverse process (TP). Twenty-eight cases of left side approach and 21 cases of right side one were performed.

\section{General preparation}

The patient was placed in the prone position over the radiolucent chest frame of the operating table. The patient's head and the upper back were placed somewhat lower than the back and buttock for sufficient circulation with 90-100 mm Hg of systolic blood pressure to decrease small bleeds. Joint arthroscopic system was used except a saline pump and an arthroscope (diameter, $4.0 \mathrm{~mm}$; zerodegree view). Saline was infused under natural gravity with 50-60 $\mathrm{cm}$ higher from the patient's back. Some kinds of BESS instruments such as angled curettes and chisels could be useful (Fig. 1).

\section{Making a basecamp}

Portals for approaching to the L5-S1 level on the skin is located at the L4-5, half proximal from the L5-S1 level to reach there tangentially (Fig. 2). On the right-side ap-

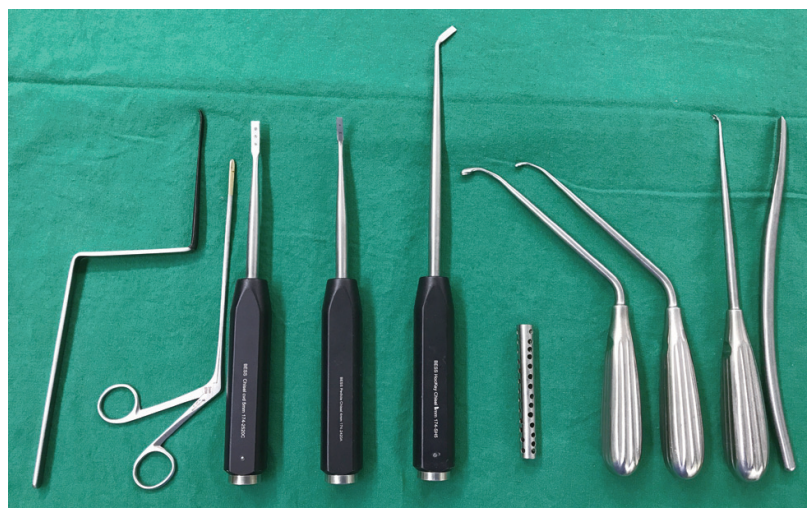

Fig. 1. BESS instrument set. The following instruments are shown from left to right: muscle detacher; 2-mm-headed, 3-mm-headed, and 5-mm-headed angled curettes; Indian Knife; 5-mm-headed osteotome; hockey chisel; and BESS-cannula. BESS, biportal endoscopic spine surgery. proach, a proximal working portal on the skin located in the middle of the TPs of the L4 and L5 and a distal viewing portal, just on the lower margin of the L5 TP with about $2 \mathrm{~cm}$ apart from the pedicular lateral margin under fluoroscopic view. On the left-side, a proximal viewing portal located in the middle of the L5 TP and a distal portal, $1 \mathrm{~cm}$ distal and $1 \mathrm{~cm}$ medial from the proximal one to avoid confronting against the iliac crest. A triangular point lateral from the two portals, i.e., quarter-back portal, could be used as an accessory portal to maintain fluent saline outflow in patients with thicker back muscles, or to enable the surgeon to reach the inner side (lateral recess) from the far outside of the foramen.

About 1-cm skin incision was enough for each portal with cross-cutting of the superficial muscle fascia for fluent saline output. The muscle detacher must go straight down and touch the dorsal surface of the TP. The basal muscle on the dorsal surface of the L5 TP and proximal surface of the alar should be scratched forcefully first using a muscle detacher before inserting a scope to infuse saline to flow-up the muscles and make a free room for scoping. Inserting a scope with a saline infusion lifted detached muscles and shaking a scope to and fro several times could help evacuating hematoma over the TP and making a clear surgical view just on the exposed bony surface of the TP (basecamp). Debridement of the rugged muscle fibers performed using a shaver, and coagulation of small bleeds using 3-mm-headed radiofrequency coagulation. If saline outflow was poor, the subcutaneous fascia of the working portal should be punched out somewhat wider using a Kerrison punch and loosened for cur-

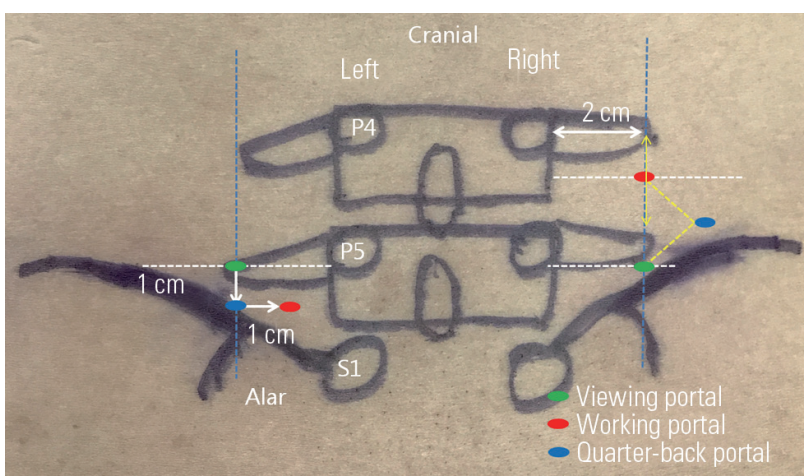

Fig. 2. Portals for the $L 5-S 1$ foraminal approach. The right side portals are somewhat different from those of the left side because of lefthanded scoping and the position of the alar wing. Lateral distance is located on about $2 \mathrm{~cm}$ from the lateral margins of the pedicles in a C-arm view. TP, transverse process. 
rent saline flow. In case of the patient with heavier back muscles as an interrupting barrier from running of saline outflow, a BESS-cannula through the quarter-back portal could be made for backing out of saline flow fluently and securing a clear view.

\section{View and orientation}

After inserting a scope over the L5 TP, its bony margin should be secured rather than the foramen itself to get an orientation. Scoping upward (12 o'clock direction) met white and smooth soft tissue (L5-S1 joint capsule). Scratching using an angled curette and shavering off the proximal part of the capsule exposed the tip of superior articular process (SAP). The foramen entrance (medially), under-surface of SAP (dorsally), and inter-TP ligament (basally) could be differentiated with a correct orientation. Carefulness should be taken not to go farther over the inter-TP ligament ventrally where there was psoas muscle.

\section{Foraminotomy, flavectomy, and discectomy}

Resection of the hypertrophied tip of SAP was performed using a hockey chisel and a Kerrison punch (Fig. 3). The tip should be resected piece by piece rather than one block. It looked a curved block so that it could not easily removed back in a very little space if it were cracked

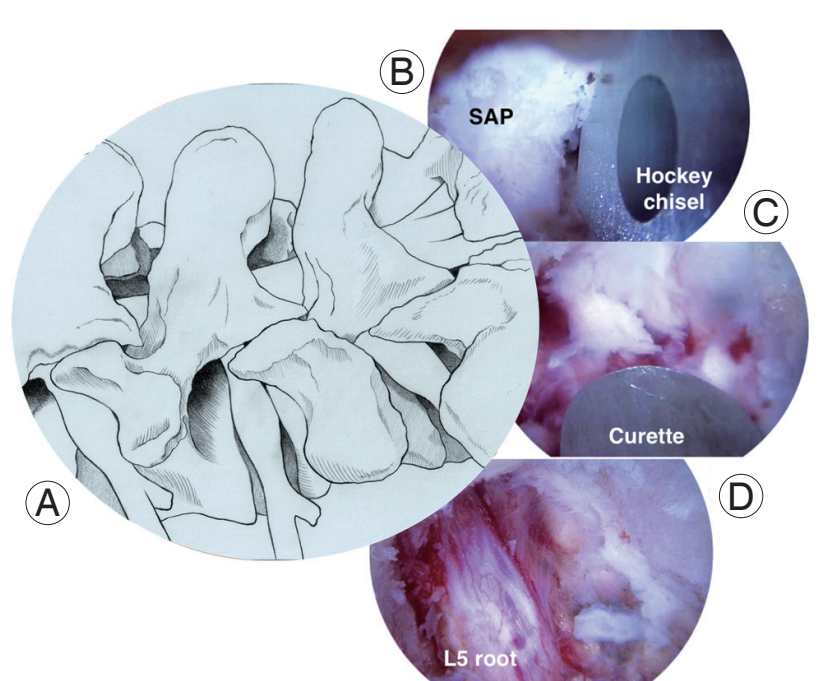

Fig. 3. Steps of the L5-S1 foraminal approach. (A) Target lesion of the overgrowing spur of the SAP. (B) Partial facetectomy of the SAP using a hockey chisel. (C) Foraminal flavectomy is performed using a curette. (D) The L5 exiting root is exposed wide enough with distal-free space about the same width of the root. SAP, superior articular process. as one large piece. The proximal margin of the foraminal ligament flavum detached from the distal surface of the L5 TP and L5 pedicle. Care should be taken not to injure the L5 exiting root just beneath the distal margin of the L5 TP. Sometime it was hidden under the L5 TP and beneath a degenerative, flabby, fibrous vascular bundle which might easily misunderstand as an atrophic, thin root. Discectomy, if needed, was performed after locating the root margin with controlling heavy bleeds from small vessels overlying the root. The outer layer or the annulus was incised using an Indian knife, and an angled curette was inserted just beneath the outer layer of the annulus to remove ruptured disc fragments, especially those under the root and far laterally at the crossing point between the root and the annulus. After sufficient decompression of the L5 root at the foramen with partial resection of the SAP, there should be an enough room with the same width of the root at the distal from the root to prevent restenosis by dynamic compression [3].

\section{Discussion}

BESS uses two portals unilaterally, so called unilateral biportal approach. That is a quietly different point from a previous report on bilateral biportal approach by Kambin et al. [4].

There must be several technical pitfalls and complications in learning curve of the new skill, such as muscle edema, hydroperitoneum, incomplete decompression, blurred surgical view, and injuring of radicular artery due to unfamiliar or misunderstanding of the anatomic structures.

Muscle edema occurred due to a poor saline output. Cross-cutting or punching-out of the superficial muscle fascia could improve outflow. Inserting a BESS-cannula through the quarter-back portal could be also helpful in patients with obesity or heavier back muscle.

If orientation were lost without scoping upward from the L5 TP and going straight down under the level of the L5 TP, the psoas muscle layer could be penetrated and infused saline could make hydroperitoneum (Fig. 4). Don't forget the direction of scoping with a very steep angle. Sono-guided paracentesis was performed to treat and aspirated the infused saline.

Under higher magnification the L5 exiting root could seem to be free after removing the foraminal ligament. However, when the patient sited and stood the foramen 

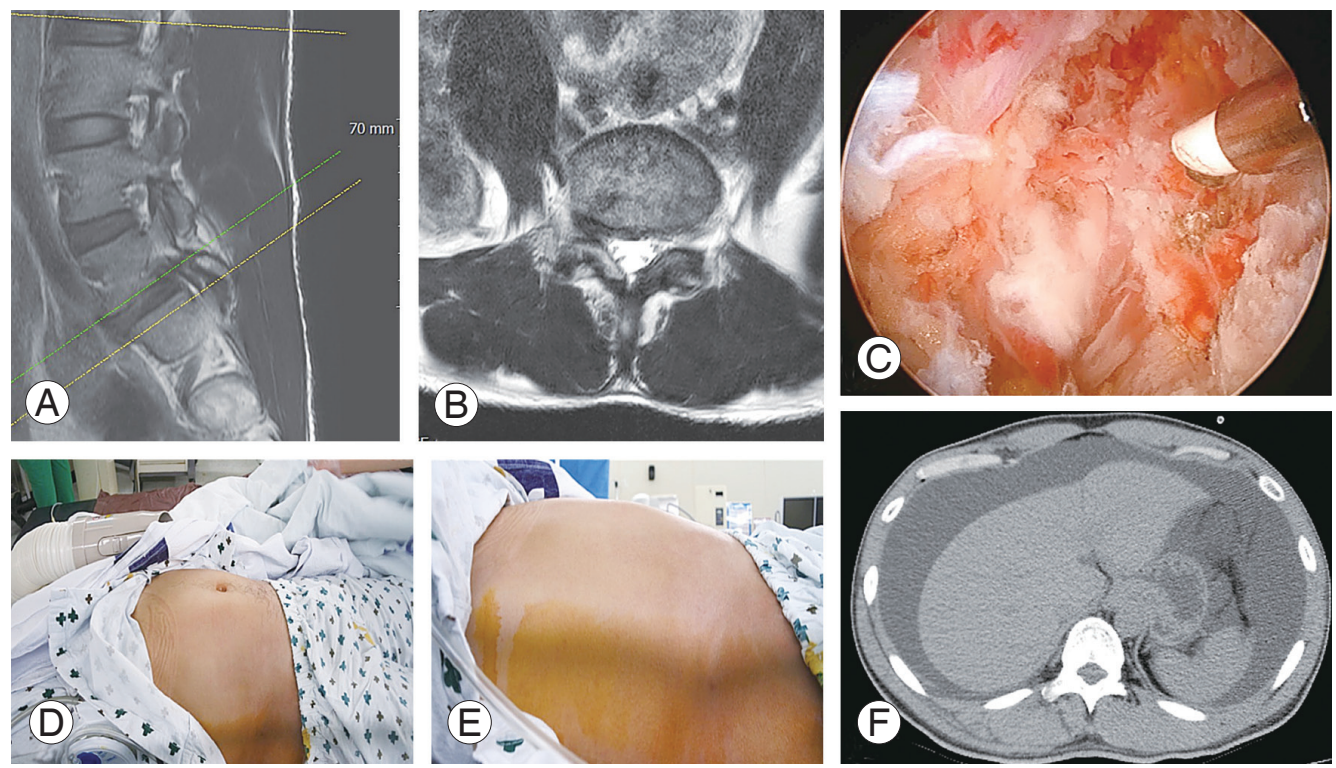

Fig. 4. Hydroperitoneum. (A, B) Preoperative magnetic resonance images showed foraminal disc herniation at the level of L5-S1. (C) Intraoperative arthroscopic view showed too much exposed ventral muscle layer. (D, E) Distended patient's abdomen in supine position. (F) Abdominal computed tomography revealed hydroperitoneum with lower intensity.

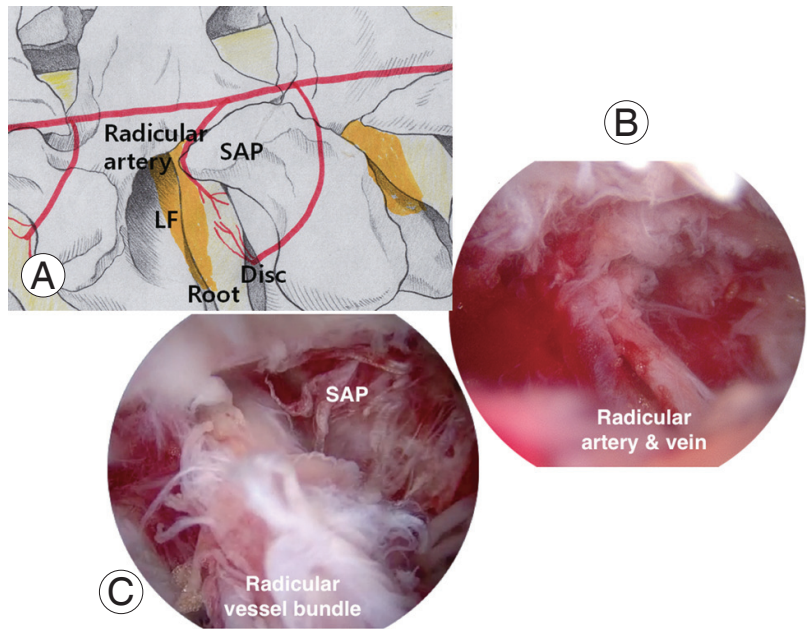

Fig. 5. Schematic anatomy of the foraminal structures. (A) The radicular artery is located over the foraminal LF. The L5 root and disc are covered and protected by the LF. (B) The radicular artery and vein are running together and looked like a vessel bundle. (C) The radicular vessel bundle is running into the just proximal to SAP. LF, ligament flavum; SAP, superior articular process.

could become narrower dynamically. The hypertrophied tip of the SAP should be removed sufficiently to decompress the root and prevent dynamic stenosis at the foramen.

Controlling the small bleeds from the vessels and radicular artery was very important to make a clear surgical field and not to injure the root. A blurred surgical field due to small bleedings interrupted the procedure frequently. Small vascular bleeding could be controlled using $1.5-\mathrm{mm}$ radiofrequency coagulation under the very lowest setting of energy with little retraction of muscle to pull the vessel backward at the time of currency firing.

The radicular artery was located at the superior side of the foramen (Fig 5). It was always encountered in the left-side approach with using left proximal scoping. In the right-side approach with left distal scoping, it was hidden under the soft tissue, and only heavy blood flow showed when its wall was scratched. An innominated artery far lateral and just proximal to the alar should be taken care of not to be injured.

In the case of spondylolytic spondylolisthesis, four compressing lesions should be decompressed completely: a spur overlying the L5 exiting root from a defected pedicle base, hypertrophied ligamentum flavum, loose body at the isthmic defect of the lamina and, and buckled and/or extruded foraminal disc. The inside approach from the far lateral side of the foramen was feasible due to hypermobility of the lamina. The foramen came to be wider when the lamina was pushed medially by levering of a scope, and the inner side could be inspected well. If the lesion inside was approached somewhat limitedly from a working portal, a quarter-back portal inserting an instrument 
with lower inclination and somewhat farther approaching medially is used.

Technically extra-foraminal approach using BESS can be surely feasible. The postoperative radiological examination matched also with patients' improvement from stenosing radicular symptoms. BESS is supposed to be an alternative treatment for foraminal decompression in various foraminal stenosing conditions with preserving the motion and stability of the L5-S1 facet and decrease the need of fusion surgery to relive various lumbar degenerative diseases.

\section{Conflict of Interest}

No potential conflict of interest relevant to this article was reported.

\section{References}

1. Matsumoto M, Watanabe K, Ishii K, et al. Posterior decompression surgery for extraforaminal entrapment of the fifth lumbar spinal nerve at the lumbosacral junction. J Neurosurg Spine 2010;12:72-81.

2. Choi DJ, Choi CM, Jung JT, Lee SJ, Kim YS. Learning curve associated with complications in biportal endoscopic spinal surgery: challenges and strategies. Asian Spine J 2016;10:624-9.

3. Kang K, Rodriguez-Olaverri JC, Schwab F, Hashem J, Razi A, Farcy JP. Partial facetectomy for lumbar foraminal stenosis. Adv Orthop 2014;2014:534658.

4. Kambin P, O'Brien E, Zhou L, Schaffer JL. Arthroscopic microdiscectomy and selective fragmentectomy. Clin Orthop Relat Res 1998;(347):150-67. 